\title{
Quantum Virtual Cooling
}

Jordan Cotler, ${ }^{1, *}$ Soonwon Choi, ${ }^{2}$ Alexander Lukin, ${ }^{3}$ Hrant Gharibyan, ${ }^{1}$ Tarun Grover, ${ }^{4}$ M. Eric Tai, ${ }^{3}$ Matthew Rispoli, ${ }^{3}$ Robert Schittko, ${ }^{3}$ Philipp M. Preiss, ${ }^{3,5}$ Adam M. Kaufman, ${ }^{3,6}$ Markus Greiner, ${ }^{3}$ Hannes Pichler, ${ }^{7,3}$ and Patrick Hayden ${ }^{1}$

${ }^{1}$ Stanford Institute for Theoretical Physics, Stanford University, Stanford, California 94305, USA

${ }^{2}$ Department of Physics, University of California, Berkeley, California 94720, USA

${ }^{3}$ Department of Physics, Harvard University, Cambridge, Massachusetts 02138, USA

${ }^{4}$ Department of Physics, University of California at San Diego, La Jolla, California 92093, USA

${ }^{5}$ Physics Institute, Heidelberg University, 69120 Heidelberg, Germany

${ }^{6}$ JILA, National Institute of Standards and Technology and University of Colorado,

and Department of Physics, University of Colorado, Boulder, Colorado 80309, USA

${ }^{7}$ ITAMP, Harvard-Smithsonian Center for Astrophysics, Cambridge, Massachusetts 02138, USA

(Received 8 January 2019; revised manuscript received 25 May 2019; published 29 July 2019)

We propose a quantum-information-based scheme to reduce the temperature of quantum many-body systems and access regimes beyond the current capability of conventional cooling techniques. We show that collective measurements on multiple copies of a system at finite temperature can simulate measurements of the same system at a lower temperature. This idea is illustrated for the example of ultracold atoms in optical lattices, where controlled tunnel coupling and quantum gas microscopy can be naturally combined to realize the required collective measurements to access a lower, virtual temperature. Our protocol is experimentally implemented for a Bose-Hubbard model on up to 12 sites, and we successfully extract expectation values of observables at half the temperature of the physical system. Additionally, we present related techniques that enable the extraction of zero-temperature states directly.

DOI: 10.1103/PhysRevX.9.031013

\section{INTRODUCTION}

Quantum simulators have been proposed to understand the complex properties of strongly correlated quantum many-body systems [1-3]. Significant progress has been made in building both analog and digital quantum simulators with a variety of quantum optical systems [4-11]. A particularly successful approach is to use cold neutral atoms in optical lattices to emulate the physics of interacting electrons in solid-state systems [2,12-19]. This is exemplified by recent experimental advances that enable explorations of quantum magnetism [20-26], measurements of many-body entanglement [27-29], and studies of quantum dynamics out of equilibrium with bosonic and fermionic atoms [28,30-33].

One of the central, outstanding challenges in these experiments is to reach the low temperatures needed to access strongly correlated phases. A prominent example is

*jcotler@stanford.edu

Published by the American Physical Society under the terms of the Creative Commons Attribution 4.0 International license. Further distribution of this work must maintain attribution to the author(s) and the published article's title, journal citation, and DOI.
Subject Areas: Atomic and Molecular Physics,

Quantum Information,

Statistical Physics given by the doped Fermi-Hubbard model with cold atoms, where small energy scales lead to correspondingly stringent temperature requirements [2]. Even though recent progress in reducing temperatures (e.g., via entropy redistribution techniques [26,34-36]) allows current quantum simulators to compete with the most advanced quantum Monte Carlo algorithms on classical computers [2], the observation of extremely low-temperature phenomena such as $d$-wave superconductivity remains elusive. This calls for the development of new techniques to reduce temperatures in quantum simulators.

In this work, we develop a novel approach to address this issue by introducing a measurement scheme that enables us to access system properties at fractions of its actual temperature $T$. Importantly, our approach achieves this without the need to physically cool the system. Instead, our "virtual" cooling protocol to a temperature $T_{\text {virtual }}=T / n$ $(n=2,3, \ldots)$ is facilitated by joint measurements on $n$ copies of the system at temperature $T$. For a schematic illustration, see Fig. 1(a). Our method can thus be used to virtually reduce the temperature of a system after all available physical cooling methods have been deployed.

Further, we detail implementations tailored to cold-atom systems in optical lattices and illustrate our protocol in an experimental quantum simulation of the Bose-Hubbard 
(a)

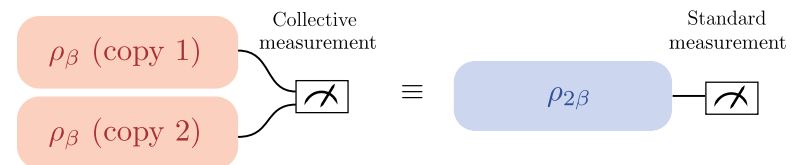

(b)

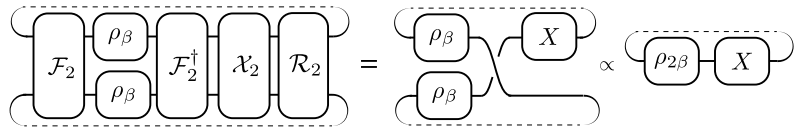

FIG. 1. (a) Schematic representation of the virtual cooling protocol. Collective measurements on two copies of a thermal state $\rho_{\beta}$ at temperature $T=1 /\left(k_{B} \beta\right)$ correspond to standard measurements at half the temperature, $T / 2$. (b) Diagrammatic representation. Two copies are evolved with the unitary $\mathcal{F}_{2}$, and a subsequent measurement of $\mathcal{X}_{2}$ and $\mathcal{R}_{2}$ is performed. In combination, this gives the expectation value $\operatorname{tr}\left\{\rho_{\beta} \rho_{\beta} X\right\} \propto$ $\operatorname{tr}\left\{\rho_{2 \beta} X\right\}$ corresponding to half the original temperature. We can also measure the proportionality constant with a similar procedure.

model. Finally, we show how these ideas can be generalized and discuss protocols to distill the many-body ground state from multiple copies of thermal many-body states.

\section{THEORETICAL OVERVIEW}

In this section, we present the theoretical ideas behind quantum virtual cooling, and we discuss experimental implementation in the following sections. We are interested in quantum many-body systems described by a thermal state $\rho(T)=e^{-\beta H} / Z$, where $H$ is the Hamiltonian of the system and $Z(T)=\operatorname{tr}\left\{e^{-\beta H}\right\}$ is the partition function at inverse temperature $\beta=1 /\left(k_{B} T\right)$. The measurement of an observable $X$ in the state $\rho$ gives the expectation value $\langle X\rangle_{T}=\operatorname{tr}\{X \rho\}$. Below, we discuss a protocol that allows us to effectively measure $\langle X\rangle_{T / n}$. The central idea is based on the ability to express the thermal density operator at $T / n$ by the $n$th power of $\rho(T)$ :

$$
\rho(T / n)=\rho(T)^{n} / \operatorname{tr}\left\{\rho(T)^{n}\right\} .
$$

In order to access the higher powers of the thermal state, we require $n$ copies of the state $\rho(T)$ prepared in parallel as well as the capability to implement operations that exchange the $n$ copies. More specifically, we have $\operatorname{tr}\left\{X \rho^{n}\right\}=\operatorname{tr}\left\{X_{s} S_{n} \rho^{\otimes n}\right\}$, where $S_{n}$ cyclically permutes quantum states in the $n$ copies, i.e., $S_{n}\left|\psi_{1}\right\rangle \otimes\left|\psi_{2}\right\rangle \otimes \ldots \otimes\left|\psi_{n}\right\rangle=\left|\psi_{2}\right\rangle \otimes\left|\psi_{3}\right\rangle \otimes \ldots \otimes\left|\psi_{1}\right\rangle$, and $X_{s}$ is the symmetrized embedding of $X$ on the $n$-fold replicated Hilbert space $X_{s}=(1 / n) \sum_{m=1}^{n} S_{n}^{m} \times$ $\left(X \otimes \mathbf{1}^{\otimes(n-1)}\right) S_{n}^{m \dagger}$. Therefore, the virtual measurement of $\langle X\rangle_{T / n}$ at temperature $T / n$ via Eq. (1) can be reduced to determining the expectation values $\left\langle X_{s} S_{n}\right\rangle$ and $\left\langle S_{n}\right\rangle$ on the $n$ copies of the state at temperature $T$. This is illustrated in Fig. 1(b). Measurements of expectation values of $S_{n}$ can be achieved with auxiliary qubits $[37,38]$, or directly via manybody state interferometry [39-41], as recently demonstrated with cold atoms [27]. We also note that our protocols apply to subsystems which are thermal, even if the global system is not thermal. In our experiments below, we leverage "eigenstate thermalization" [42-46] to obtain thermal reduced density matrices from globally pure states of finite energy density in a chaotic system. Earlier theoretical work provided numerical evidence that a chaotic eigenstate or a reduced density matrix of a thermal state encodes correlations at all temperatures $[47,48]$.

Below, we discuss protocols to measure $\left\langle X_{s} S_{n}\right\rangle$ for arbitrary $n$ and detail the procedure for the simplest example, $n=2$. We first focus on an interferometric measurement scheme and demonstrate that it can be implemented in current experiments with cold atoms. Alternative virtual cooling schemes using ancillary atoms are discussed below. Finally, we show that schemes with ancillary atoms can be generalized to not only virtually cool a many-body system, but directly distill and prepare the many-body ground state from a thermal state. Importantly, all of the discussed protocols are agnostic to the temperature $T$ of the physical system, and thus can be used to obtain additional, virtual cooling even after all available physical cooling methods have been deployed.

\section{INTERFEROMETRIC MEASUREMENT}

To simplify the presentation we first discuss a virtual cooling scheme for bosonic atoms in optical lattices. The key idea is to represent the permutation operator $S_{n}$ in the bosonic Hilbert space as $S_{n}=\mathcal{F}_{n}^{\dagger} \mathcal{R}_{n} \mathcal{F}_{n}$, where the unitary $\mathcal{F}_{n}$ denotes the discrete Fourier transformation,

$$
\mathcal{F}_{n} a_{p, j} \mathcal{F}_{n}^{\dagger}=\frac{1}{\sqrt{n}} \sum_{k=1}^{n} e^{i(2 \pi k p / n)} a_{k, j}
$$

and $\mathcal{R}_{n}=\prod_{j} e^{-i 2 \pi / n \sum_{p=1}^{n} p n_{p, j}}$ [49]. Here, $a_{p, j}$ denotes the bosonic annihilation operator on site $j$ in copy $p$, and $n_{p, j}=a_{p, j}^{\dagger} a_{p, j}$ is the corresponding number operator. Note that $\mathcal{F}_{n}$ can be realized by simply introducing tunnel coupling between neighboring copies [40], and $\mathcal{R}_{n}$ can be directly measured with a number-resolving quantum gas microscope. This representation of the permutation operator suggests that we introduce an operator $\mathcal{X}_{n}=\mathcal{F}_{n} X_{s} \mathcal{F}_{n}^{\dagger}$, which is the discrete Fourier transform of the observable $X$ that we want to measure. With this definition we can express

$$
\langle X\rangle_{T / n}=\operatorname{tr}\left\{\mathcal{X}_{n} \mathcal{R}_{n}\left(\mathcal{F}_{n} \rho^{\otimes n} \mathcal{F}_{n}^{\dagger}\right)\right\} / \operatorname{tr}\left\{\mathcal{R}_{n}\left(\mathcal{F}_{n} \rho^{\otimes n} \mathcal{F}_{n}^{\dagger}\right)\right\} .
$$

A measurement of $X$ at the virtually reduced temperature $T / n$ thus consists of a measurement of $\mathcal{X}_{n} \mathcal{R}_{n}$ and $\mathcal{R}_{n}$ after application of the discrete Fourier transform across the copies. For many interesting observables one finds 
$\left[\mathcal{X}_{n}, \mathcal{R}_{n}\right]=0$, so that $\mathcal{R}_{n}$ and $\mathcal{X}_{n}$ can be measured independently.

As a specific example, we consider the experimentally simplest case $n=2$ and the measurement of the on-site density by choosing $X \equiv n_{j}$. The corresponding protocol consists of three steps. (i) We prepare $n=2$ identical instances of the thermal many-body state $\rho(T)$. This can be achieved, for example, by preparing two identical states in neighboring $1 \mathrm{D}$ tubes, or 2D planes. It is essential that the copies are decoupled at this stage, which can be achieved by using a large optical potential between the tubes or planes to suppress any intercopy tunneling. (ii) We then freeze the dynamics within each copy, and lower the potential between the two copies, e.g., using an optical superlattice. This induces tunneling between the two copies via the Hamiltonian $H_{\mathrm{BS}}=-J_{\mathrm{BS}} \sum_{j}\left(a_{1, j}^{\dagger} a_{2 j}+\right.$ H.c. $)$, which allows us to realize the so-called beam splitter operation $\mathcal{F}_{2}$ that maps $\rho^{\otimes 2} \rightarrow \mathcal{F}_{2} \rho^{\otimes 2} \mathcal{F}_{2}^{\dagger}$. Interactions between the atoms need to be turned off (e.g., via a Feshbach resonance) or made negligible as compared to

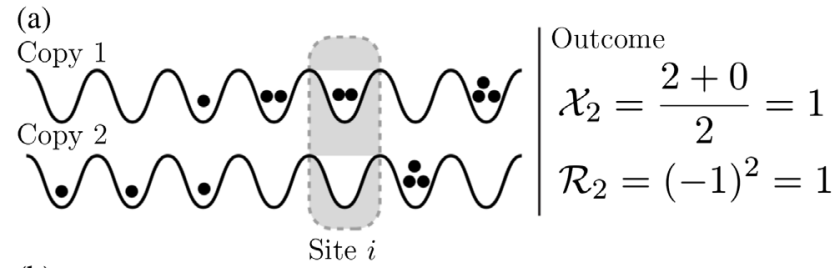

(b)

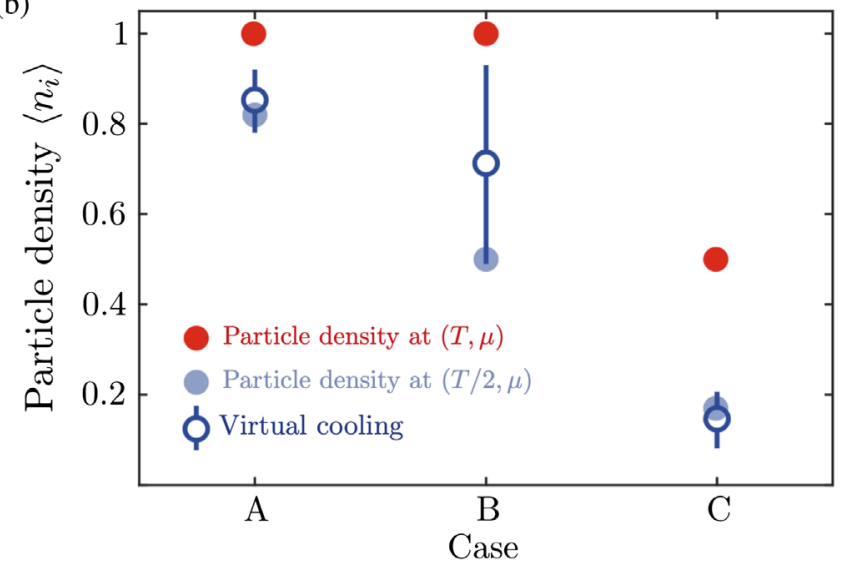

FIG. 2. (a) Schematic for a single measurement trial of $\mathcal{X}_{2}=$ $\frac{1}{2}\left(n_{1, i}+n_{2, i}\right)$ and $\mathcal{R}_{2}$ restricted to the $i$ th site, after $\mathcal{F}_{2}$ has been applied to the two copies. (b) Measured single-site density, averaged over all but the edge sites of the chain, after virtual cooling has been applied to the system (blue circles with vertical error bars). Red disks show the single-site density of the state before our protocols are utilized (the actual density of particles in each experiment), whereas light blue disks correspond to the prediction of the effective thermal ensemble (see text) at half the temperature. Agreement of the data with the reduced-temperature ensemble validates the applicability of our method in the experimental system. Error bars denote the standard error of the mean.
$J_{\mathrm{BS}}$ during this step. (iii) Finally, we measure the on-site occupation number on all sites in both copies using a numberresolving quantum gas microscope. This gives direct access to $\mathcal{R}_{2}=(-1)^{\sum_{j} n_{1, j}}$ and $\mathcal{X}_{2}=\mathcal{F}_{2} \frac{1}{2}\left(n_{1, j}+n_{2, j}\right) \mathcal{F}_{2}^{\dagger}=$ $\frac{1}{2}\left(n_{1, j}+n_{2, j}\right)$. Averaging the results over multiple experiments gives the expectation value of the local density at $T / 2$ via Eq. (3) [for a schematic of a single measurement trial, see Fig. 2(a)]. Remarkably, this experimental procedure parallels the one employed to determine the second-order Rényi entropy of cold atoms, with atom number-resolved measurements being the only additional requirement. Such measurements were first demonstrated for one-dimensional systems using full-atom-number-resolved imaging in a quantum gas microscope [28].

\section{EXPERIMENTAL DEMONSTRATION}

In order to demonstrate our protocol, we experimentally realize it in a one-dimensional Bose-Hubbard model. In the experiment, a Bose-Einstein condensate of ${ }^{87} \mathrm{Rb}$ atoms is loaded into a two-dimensional optical lattice positioned at the focus of a high-resolution imaging system. The dynamics of the atoms is well described by a Bose-Hubbard Hamiltonian parametrized by tunneling strength $J$ and onsite interaction energy $U$ (see Ref. [28] for details).

The experimental protocol consists of four steps: initialization, quenched thermalization dynamics, beam splitter operations, and measurements. During initialization, optical potentials are sequentially manipulated in order to isolate an initial product state $\left|\psi_{0}\right\rangle$ with a single atom on the central $2 \times 6$ sites of a $2 \times L$ plaquette in the deep $45 E_{r}$ lattice where the tunneling between the sites is negligible [28]. Each $1 \times L$ tube represents an identical copy of the system. Next, the lattice potential along the chains is suddenly lowered, allowing particles to tunnel and interact within each chain. It has been previously shown [28] that this quenched dynamics drives the thermalization of small subsystems within the chain. Hence, after sufficiently long time evolution, the state of the subsystem can be described by an effective temperature $T$ and chemical potential $\mu$, which are determined by the total energy and particle number density of $\left|\psi_{0}\right\rangle$. (See also Ref. [48].) After the desired time evolution, the dynamics of the system is frozen by suddenly increasing the lattice depth along the chains, and a beam splitter operation $\mathcal{F}_{2}$ is implemented by lowering the potential barrier between the two chains, such that particles can tunnel (in the transverse direction) for a prescribed time. Finally, the number of particles on each individual lattice site is measured. This procedure is repeated multiple times in order to obtain sufficient statistics.

We apply our virtual cooling protocol in three regimes (A, B, and C), with differing initial states $\left|\psi_{0}\right\rangle$, system size $L$, and Hamiltonian parameters $U / J$. For the datasets $\mathrm{A}$ and $\mathrm{B}$, each of $L=6$ sites is initially occupied by one particle, 
whereas for the dataset $\mathrm{C}$, only the middle six out of the total $L=12$ sites are occupied by one particle per site. The tunneling rates are set such that $U / J \approx 1.56$ (dataset $\mathrm{A}$ ) or 0.33 (datasets $\mathrm{B}$ and $\mathrm{C}$ ). These combinations lead to the effective temperatures and chemical potentials $(T / J, \mu / J) \approx(3.5,-1.0),(11.5,-6.3)$, and $(18.3,-17.7)$ of subsystems for datasets A, B, and C, respectively. Based on our protocol, we extract the average particle number density $\left\langle n_{i}\right\rangle$ of the $i$ th site for thermal ensembles at reduced temperature.

Figure 2(b) shows the resulting single-site particle density after virtual cooling for all three cases. We compare these results with the initial single-site density at the original temperatures as well as theoretical predictions from an ideal thermal ensemble $\rho_{2 \beta}$ at half of the original temperatures. All data points are in good agreement with the reduced temperature ensemble, indicating that our virtual cooling scheme works in the experimental system.

\section{OBSERVABLES}

The protocol presented in Sec. III allows us to measure local densities at reduced temperatures. In this section, we discuss some of the issues that arise when generalizing this scheme to more complicated observables and present an alternative protocol that avoids these issues.

One of the useful properties of the single-site density $X \equiv n_{j}$ is that its symmetrized version $X_{s}=\frac{1}{2}\left(n_{1, j}+n_{2, j}\right)$ is invariant under conjugation by $\mathcal{F}_{2}$, i.e., $\mathcal{X}_{2}=$ $\mathcal{F}_{2} X_{s} \mathcal{F}_{2}^{\dagger}=\frac{1}{2}\left(n_{1, j}+n_{2, j}\right)$. Thus, $\mathcal{X}_{2}$ is easily measured by averaging the number of atoms on the $j$ th site in the two copies. Furthermore, $\mathcal{X}_{2}$ commutes with $\mathcal{R}_{2}$, and so we can measure the observables in either order. In fact, $\mathcal{X}_{2}$ and $\mathcal{R}_{2}$ commute with all single-site densities $n_{1, j}, n_{2, k}$, and so we can simply measure the individual particle numbers and combine them to compute the expectation values of $\mathcal{X}_{2}$ and $\mathcal{R}_{2}$.

For more complicated observables such as densitydensity correlators $X \equiv n_{j} n_{\ell}$, the situation is more subtle. A direct application of the procedure outlined above requires a measurement of

$$
\begin{aligned}
\mathcal{X}_{2}= & \mathcal{F}_{2} \frac{1}{2}\left(n_{1, j} n_{1, \ell}+n_{2, j} n_{2, \ell}\right) \mathcal{F}_{2}^{\dagger} \\
= & \frac{1}{4}\left(n_{1, j}+n_{2, j}\right)\left(n_{1, \ell}+n_{2, \ell}\right) \\
& +\frac{1}{4}\left(a_{1, j}^{\dagger} a_{2, j}+a_{2, j}^{\dagger} a_{1, j}\right)\left(a_{1, \ell}^{\dagger} a_{2, \ell}+a_{2, \ell}^{\dagger} a_{1, \ell}\right) .
\end{aligned}
$$

While the first term in Eq. (4) (i.e., the final equality) is easily measurable with standard quantum gas microscopy, the second term requires an additional interferometric apparatus.

Before proceeding with the discussion of an alternative protocol that avoids this issue (see Sec. VA), let us note that the first term of Eq. (4) by itself contains interesting information about the system at half of its temperature. This first term of Eq. (4) is easy to measure, since it commutes with $\mathcal{R}_{2}$ and all of the number operators. Doing so would output the unconventional correlator:

$$
\frac{1}{2} \operatorname{tr}\left\{n_{j} n_{\ell} \rho(T / 2)\right\}+\frac{1}{2} \frac{\operatorname{tr}\left\{n_{j} \rho(T) n_{\ell} \rho(T)\right\}}{\operatorname{tr}\left\{\rho(T)^{2}\right\}} .
$$

The term on the left-hand side here is the desired equal-time density-density correlator at half the system temperature, whereas the term on the right-hand side is peculiar. In fact, this peculiar term is equal to the unequal imaginarytime correlator $\frac{1}{2} \operatorname{tr}\left\{n_{j}(1 / T) n_{\ell} \rho(T / 2)\right\}$, where $n_{j}(\tau)=$ $e^{H \tau} n_{j} e^{-H \tau}$ is the number density evolved in imaginary time. If our system is translation invariant and at sufficiently low temperature, we expect $\operatorname{tr}\left\{n_{j} n_{\ell} \rho(T / 2)\right\}$ to depend on $|j-\ell|$, whereas the peculiar term should not strongly depend on $|j-\ell|$. This is because at low temperatures the large imaginary time evolution of the operator $n_{j}$ scrambles it strongly, destroying the memory of its initial position $j$. Indeed, in the limit of $T \rightarrow 0$, the peculiar term is just $\left\langle\psi_{0}\left|n_{j}\right| \psi_{0}\right\rangle\left\langle\psi_{0}\left|n_{\ell}\right| \psi_{0}\right\rangle$, which is clearly independent of $|j-\ell|$. At high temperature and small $|j-\ell|$, both terms in Eq. (5) have a nontrivial dependence on $|j-\ell|$, and so we are unable to extract each term separately. Nevertheless, it is interesting to note that our protocol yields some information about the unequal imaginary-time correlator in this regime. We note also that when $|j-\ell|$ is much larger than the thermal correlation length, both terms in Eq. (5) approach $\frac{1}{2} \operatorname{tr}\left\{n_{j} \rho(T / 2)\right\} \operatorname{tr}\left\{n_{\ell} \rho(T / 2)\right\}$. We explore the dependence of $\operatorname{tr}\left\{n_{j} \rho(T) n_{\ell} \rho(T)\right\}$ on $|j-\ell|$ as a function of $T$ in the Appendixes and confirm that there is essentially no dependence at sufficiently low temperatures.

\section{A. Ancilla qubit approach to cooling}

The example above shows that for some observables a direct measurement of the $\mathcal{F}_{2}$ conjugation may be challenging. Here, we present an alternative approach that is experimentally feasible.

Consider a nondestructive measurement of the SWAP operator $S_{2}$ on two systems which are each prepared in the state $\rho$. Since $S_{2}$ is unitary and Hermitian, the two possible measurement outcomes are \pm 1 , corresponding to projections into the symmetric or antisymmetric subspace with respect to the exchange of the two copies. The state after such a measurement is thus given by $\mathcal{P}_{ \pm}(\rho \otimes \rho) \mathcal{P}_{ \pm} / \operatorname{tr}\left\{\mathcal{P}_{ \pm}(\rho \otimes \rho)\right\}$, with $\mathcal{P}_{ \pm}=\left(1 \pm S_{2}\right) / 2$. If the measurement outcome is -1 , we discard both systems. But for those instances that yield a measurement +1 , we retain one of the systems and discard only the other one. The resulting state of this first system $\rho_{1}$ is obtained by tracing out the degrees of freedom of the second system: 


$$
\rho_{1}=\frac{\operatorname{tr}_{2}\left\{\mathcal{P}_{+}(\rho \otimes \rho) \mathcal{P}_{+}\right\}}{\operatorname{tr}\left\{\mathcal{P}_{+}(\rho \otimes \rho)\right\}}=\frac{\rho+\rho^{2}}{1+\operatorname{tr}\left\{\rho^{2}\right\}}
$$

For an initial thermal state $\rho(T)$, the new state $\rho_{1}$ corresponds to a mixture of $\rho(T)$ and $\rho(T / 2)$. The success probability for achieving $\rho_{1}$ is $p_{+}=\left(1+\operatorname{tr}\left\{\rho^{2}\right\}\right) / 2$, which is always larger than $1 / 2$.

Now to measure $\operatorname{tr}\{X \rho(T / 2)\}$, we first measure $\operatorname{tr}\{X \rho(T)\}$ and then $\operatorname{tr}\left\{X \rho_{1}\right\}$. Through the process of measuring $X$ with respect to $\rho_{1}$, we automatically determine $p_{+}$. Then we put together our measurements as

(a)

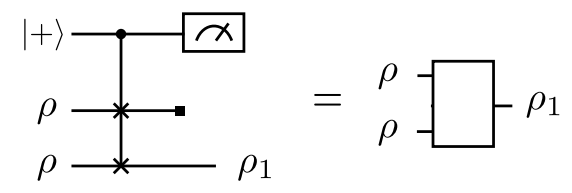

(b)

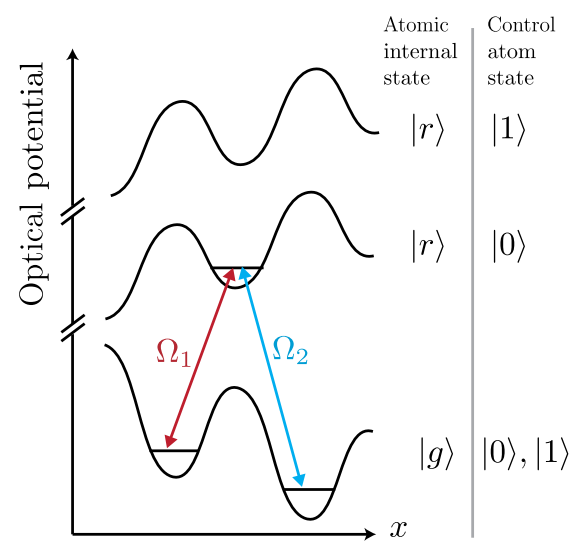

(c)

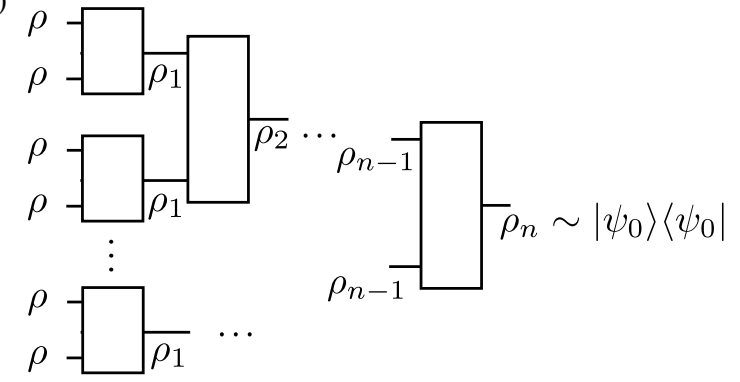

FIG. 3. (a) Quantum circuit representation of ancilla qubit virtual cooling protocol. Following the controlled SWAP of two copies of a quantum state, the control ancilla qubit is measured. If the qubit is measured to be $|0\rangle$, then one of the system copies is discarded and the remaining system copy is in the state $\rho_{1}$. (b) The controlled SWAP operation can be implemented for ultracold atoms on an optical lattice by the combination of photon-assisted hopping and the Rydberg blockade mechanism; excitation of the control atom in a Rydberg state conditionally prevents photon-assisted hopping. (c) Quantum circuit representation of ground-state distillation protocol. The protocol in (a) can be parallelized and nested, as shown in the diagram. If $\rho$ is a thermal state, then the ground state will be distilled.

$$
\frac{2 p_{+}}{2 p_{+}-1} \operatorname{tr}\left\{X \rho_{1}\right\}-\frac{1}{2 p_{+}-1} \operatorname{tr}\{X \rho(T)\}=\operatorname{tr}\{X \rho(T / 2)\},
$$

which gives us the desired measurement of $\rho(T / 2)$.

Nondestructive measurements of the SWAP operator are typically challenging. One way to realize such measurements is to use ancillary qubits [37]. A nondestructive measurement of the SWAP operator can then be realized by a simple quantum circuit, in which the ancillary qubit is initially prepared in the state $(1 / \sqrt{2})(|0\rangle+|1\rangle)$, followed by the application of a controlled SWAP operation, exchanging the quantum state of the two copies conditional on the ancillary qubit being in state $|0\rangle$, and a final measurement of the ancillary qubit (see Fig. 3). (This is the opposite of the usual convention for controlled SWAP gates, but will be convenient immediately below.)

In a cold-atom setup, one can envision realizing the required controlled SWAP operations using Rydberg interactions [50]. For example, one might encode the ancillary qubit states in two internal states of an ancillary atom, with $|0\rangle$ being the internal ground state and $|1\rangle$ a highly excited, long-lived, Rydberg state. The Rydberg blockade mechanism can be used to control the tunnel coupling between two copies of an optical lattice and so realize a controlled exchange operation. To see this, consider inducing a tunnel coupling between the initially decoupled copies using a two-photon Raman process [15,16,51]. If this Raman process involves a Rydberg state as an intermediate level (see Fig. 3), it is affected by the state of the ancillary atom. In particular, if the ancillary atom is in a Rydberg state, the strong dipolar interactions can lead to a shift of the energy of this intermediate state and completely inhibit tunneling. As a consequence, the exchange of the quantum state of the two copies can be completely controlled by the ancillary atom. We note that similar protocols have been discussed and analyzed in the literature [50].

\section{GROUND-STATE DISTILLATION}

The ancilla qubit approach can be generalized to schemes that not only allow us to measure a system at reduced temperatures, but further enable the distillation of the ground state from multiple copies of a thermal ensemble. This is akin to entanglement purification proposals for quantum communication over noisy channels [52].

Consider again the protocol explained in the previous section, in which we couple $\rho \otimes \rho$ to an ancilla and obtain the state $\rho_{1}=\left(\rho+\rho^{2}\right) /\left(1+\operatorname{tr}\left\{\rho^{2}\right\}\right)$ with probability $p_{+}=\left(1+\operatorname{tr}\left\{\rho^{2}\right\}\right) / 2$. If $\rho(T)$ is a thermal state at temperature $T$, then $\rho_{1}$ is a linear combination $\rho(T)$ and $\rho(T / 2)$. Clearly, $\rho_{1}$ has the same eigenvectors as $\rho$, but with different eigenvalues. In particular, $\rho_{1}$ is purer than $\rho$, and the eigenvalue of the largest eigenvector (i.e., the ground state for thermal $\rho$ ) is larger. This purification is of 
course probabilistic, as its success is conditioned on the proper measurement outcome for $S_{2}$. Remarkably, the success probability $p_{+}=\left(1+\operatorname{tr}\left\{\rho^{2}\right\}\right) / 2$ is always larger than $1 / 2$ and approaches 1 as the system is purified. Starting with multiple copies one can iterate the above process, which will ultimately converge to a system in the largest eigenstate of $\rho$. For thermal states, the procedure distills the many-body ground state, i.e., the zero-temperature state.

\section{LIMITATIONS AND SCALABILITY}

We are often interested in local observables $X$, which in turn correspond to the local observables $X_{s}$. Suppose that $X$ is supported on a subregion $R$. Then $X_{s}$ is supported on the joint region $R_{1} \cup R_{2}$ of the two corresponding system copies. For concreteness, suppose our system is one dimensional. We desire to measure

$$
\operatorname{tr}\{X \rho(T / 2)\}=\operatorname{tr}_{R}\left\{X \rho_{R}(T / 2)\right\}
$$

where $\rho_{R}(T / 2)=\operatorname{tr}_{\bar{R}}\{\rho(T / 2)\}$ is the reduced density matrix of $\rho(T / 2)$ on $R$. Naively, it seems that we only need to perform our procedure on the subsystem $R_{1} \cup R_{2}$ of the two copies. However, this is not correct, since

$$
\frac{\rho_{R}^{2}}{\operatorname{tr}_{R}\left\{\rho_{R}^{2}\right\}} \neq \operatorname{tr}_{\bar{R}}\left\{\frac{\rho^{2}}{\operatorname{tr}\left\{\rho^{2}\right\}}\right\}=\operatorname{tr}_{\bar{R}}\{\rho(T / 2)\}
$$

Nonetheless, suppose we extend $R$ by buffering each of its boundaries by a number of sites corresponding to the correlation length of the system at temperature $T / 2$. Let us denote this extended region by $B$. Here, $R \subset B$, but $B$ is smaller than the whole system. The corresponding joint region of the two system copies is $B_{1} \cup B_{2}$. If we perform our procedure on the subsystem $B_{1} \cup B_{2}$ of the two copies, we can access the state $\sigma_{B} \equiv \rho_{B}^{2} / \operatorname{tr}_{B}\left\{\rho_{B}^{2}\right\}$, which satisfies $\sigma_{B} \approx \operatorname{tr}_{\bar{R}}\{\rho(T / 2)\}$, and therefore

$$
\operatorname{tr}_{B}\left\{X \sigma_{B}\right\} \approx \operatorname{tr}\{X \rho(T / 2)\} .
$$

So if we choose $B$ large enough (but in most cases, smaller than the size of the entire system), we can still approximately measure our desired observable.

\section{A. Scalability}

We discuss the scalability in terms of two parameters, the temperature of the total system and the size of the subsystem to be measured. In particular, we are interested in the limit where the temperature is low and the total system size is large. In an experiment, the performance of a measurement protocol is fundamentally limited by the number of repetitions required to determine the averages to sufficiently high precision. In our setting, the measurement statistics required to precisely measure the denominator $Z_{n}=\operatorname{tr}\left\{\rho(T)^{n}\right\}$ in Eq. (1) may be a limiting factor. In a many-body system, $Z_{n}$ is directly related to the Rényi- $n$ entropy $S_{n}=[1 /(1-n)] \log \left(Z_{n}\right)$, which scales with volume for local systems. $Z_{n}$ is therefore often exponentially small in the system size.

Hence, one would generally need a large number of measurements, $N_{m} \sim 1 / Z_{n}^{2} \sim \exp \{2 s(T)|R|\}$, where $s(T)$ is the entropy density at temperature $T$ and $|R|$ is the size of the subregion on which $\rho(T)$ is supported. In the limit of low temperature, this scaling becomes favorable since $s(T)$ generally decreases. However, the thermal correlation length $\xi(T / n)$ can increase as $T$ is lowered, requiring a larger subregion size, $|R| \geq \xi(T / n)$. Together, the number of measurements required to achieve some fixed precision scales as $N_{m} \sim \exp \{2 s(T) \xi(T / n)\}$. In practice, the correlation length of particular two-point functions may be smaller than the thermal correlation length, depending on the choice of operator insertions. Accordingly, a smaller, effective correlation length for particular observables yields a more favorable scaling in the number of measurements.

Of course, if $\rho$ is only approximately thermal, then expectation values of $\rho^{n} / \operatorname{tr}\left\{\rho^{n}\right\}$ for larger values of $n$ can have amplified deviations from thermality. However, if we are interested in the physics of the ground state $\left|\psi_{0}\right\rangle$, then $\rho^{n} / \operatorname{tr}\left\{\rho^{n}\right\} \sim\left|\psi_{0}\right\rangle\left\langle\psi_{0}\right|$ for larger values of $n$ so long as $\left|\psi_{0}\right\rangle$ is the dominant eigenstate of $\rho$.

\section{DISCUSSION}

Reaching low temperatures is paramount for studying interesting quantum many-body phases with quantum simulators. In particular, the small energy scales in coldatom systems pose a major challenge for accessing the required temperature regimes. In this work, we propose and demonstrate novel techniques that enable access to properties of a system at a fraction of its actual temperature. This virtual cooling is enabled by collective measurements on multiple copies of the system.

More generally, our schemes illustrate a connection between thermal physics and entanglement. In particular, the temperature of a system is intimately connected to its entanglement with its surroundings $[28,43,44,48,53,54]$. Accordingly, measuring correlations of a thermal system at virtually lower temperatures involves manipulating and probing entanglement. This is why the tools for measuring a system at virtually lower temperatures resemble those that allow access to entanglement entropies [27,39,40].

A natural future direction is to experimentally perform quantum virtual cooling for more complicated observables. A particularly interesting application would be to experimentally study a quantum many-body system with a finitetemperature phase transition at some temperature $T_{c}$. One could prepare the system at some temperature $T>T_{c}$, and use virtual cooling to probe features at or below the phase transition. (For related theoretical work, see Ref. [55].) Understanding the range of applicability of quantum virtual 
cooling is an exciting theoretical and experimental program, which will require new insights into subsystem eigenstate thermalization and thermalization more broadly.

\section{ACKNOWLEDGMENTS}

We thank Alex Avdoshkin for helpful conversations. J. C. is supported by the Fannie and John Hertz Foundation and the Stanford Graduate Fellowship program. S.C. acknowledges support from the Miller Institute for Basic Research in Science. A. L., R. S., and M. G. are supported by the NSF, the Gordon and Betty Moore Foundation's EPiQS Initiative, and the Air Force Office of Scientific Research MURI program. H. G. was supported in part by NSF Grant No. PHY-1720397. M. R. was supported by a NSF Graduate Research Fellowship. P. M. P. acknowledges funding through the ERC Consolidator Grant No. 725636 and the Daimler and Benz foundation. T. G. is supported as an Alfred P. Sloan Research Fellow. H. P. is supported by the NSF through a grant for the Institute for Theoretical Atomic, Molecular, and Optical Physics at Harvard University and the Smithsonian Astrophysical Observatory. P. H. was supported by AFOSR (FA9550-161-0082), CIFAR, the Simons Foundation, and DOE Award No. DE-SC0019380.

\section{APPENDIX A: FURTHER DETAILS OF QUANTUM VIRTUAL COOLING}

Here, we present detailed quantum virtual cooling schemes, including ones that do not appear in the main text. We analyze the case of two system copies, so that quantum virtual cooling allows us to probe observables at half of the physical temperature. In particular, we specialize to bosons and fermions in optical lattices.

\section{Boson interferometry}

If our two identical systems are bosonic, then we can perform quantum virtual cooling along the lines of the main text. In particular, we do not need an ancilla qubit to facilitate the application of the SWAP operator. Consider the bosonic Hilbert space $\operatorname{Sym}\left(\mathcal{H}_{1} \otimes \mathcal{H}_{2}\right)$, comprising of two systems with $N$ sites each. A basis for $\operatorname{Sym}\left(\mathcal{H}_{1} \otimes \mathcal{H}_{2}\right)$ is

$\left|\left\{p_{i}\right\},\left\{q_{j}\right\}\right\rangle=\prod_{i, j=1}^{N}\left(a_{2, i}^{\dagger}-a_{1, i}^{\dagger}\right)^{p_{i}}\left(a_{2, j}^{\dagger}+a_{1, j}^{\dagger}\right)^{q_{j}}|\mathrm{vac}\rangle$,

for $\left\{p_{i}\right\},\left\{q_{j}\right\} \in \mathbb{Z}_{\geq 0}^{\times N}$. From Eq. (2), $\mathcal{F}_{2}$ is a unitary which maps

$$
\begin{aligned}
& \mathcal{F}_{2} \frac{1}{\sqrt{2}}\left(a_{2, i}^{\dagger}+a_{1, i}^{\dagger}\right) \mathcal{F}_{2}^{\dagger}=a_{2, i}^{\dagger}, \\
& \mathcal{F}_{2} \frac{1}{\sqrt{2}}\left(a_{2, i}^{\dagger}-a_{1, i}^{\dagger}\right) \mathcal{F}_{2}^{\dagger}=a_{1, i}^{\dagger} .
\end{aligned}
$$

Furthermore, $\mathcal{R}_{2}=(-1)^{\sum_{j} n_{1, j}}$ is the total parity operator for the first of the two identical systems. It is easy to check that

$$
S_{2}\left|\left\{p_{i}\right\},\left\{q_{j}\right\}\right\rangle=\mathcal{F}_{2}^{\dagger} \mathcal{R}_{2} \mathcal{F}_{2}\left|\left\{p_{i}\right\},\left\{q_{j}\right\}\right\rangle,
$$

and so

$$
\operatorname{tr}\left\{\mathcal{R}_{2} \mathcal{F}_{2} \rho^{\otimes 2} \mathcal{F}_{2}^{\dagger}\right\}=\operatorname{tr}\left\{S_{2} \rho^{\otimes 2}\right\}=\operatorname{tr}\left\{\rho^{2}\right\}
$$

Then if we have an operator $X$ that we wish to measure, the idea is to instead measure $\mathcal{X}_{2}=\mathcal{F}_{2} \frac{1}{2}(X \otimes \mathbf{1}+\mathbf{1} \otimes X) \mathcal{F}_{2}^{\dagger}$, so that, in essence,

$$
\begin{aligned}
\operatorname{tr}\left\{\mathcal{R}_{2} \mathcal{X}_{2} \mathcal{F}_{2} \rho^{\otimes 2} \mathcal{F}_{2}^{\dagger}\right\} & =\frac{1}{2} \operatorname{tr}\left\{\mathcal{R}_{2} \mathcal{F}_{2}(X \otimes \mathbf{1}+\mathbf{1} \otimes X) \rho^{\otimes 2} \mathcal{F}_{2}^{\dagger}\right\} \\
& =\frac{1}{2} \operatorname{tr}\left\{S_{2}(X \otimes \mathbf{1}+\mathbf{1} \otimes X) \rho^{\otimes 2}\right\} \\
& =\operatorname{tr}\left\{X \rho^{2}\right\}
\end{aligned}
$$

Of course, there is a detailed measurement procedure that realizes the above equations.

To measure $\operatorname{tr}\left\{X \rho^{2}\right\} / \operatorname{tr}\left\{\rho^{2}\right\}$, we use the following procedure.

(1) Start with the initial state $\rho^{\otimes 2}$.

(2) Apply $\mathcal{F}_{2}$ to obtain

$$
\sum_{i} \mathcal{F}_{2} \rho^{\otimes 2} \mathcal{F}_{2}^{\dagger}
$$

(3) Measure the operator $\mathcal{X}_{2}$, given by

$$
\mathcal{X}_{2}=\mathcal{F}_{2}\left[\frac{1}{2} X\left(\left\{a_{1, i}, a_{1, i}^{\dagger}\right\}\right)+\frac{1}{2} X\left(\left\{a_{2, i}, a_{2, i}^{\dagger}\right\}\right)\right] \mathcal{F}_{2}^{\dagger} .
$$

Here, $X\left(\left\{a_{1, i}, a_{1, i}^{\dagger}\right\}\right)$ denotes that the operator is written in terms of sums of products of creation and annihilation operators in the set $\left\{a_{1, i}, a_{1, i}^{\dagger}\right\}_{i \in \text { sites }}$, and similarly for $X\left(\left\{a_{2, i}, a_{2, i}^{\dagger}\right\}\right)$. The operator $\mathcal{X}_{2}$ has the property $\left[\mathcal{X}_{2}, \mathcal{R}_{2}\right]=0$, which will be utilized shortly. Suppose $\mathcal{X}_{2}=\sum_{i} \lambda_{i} P_{i}$, where the $\left\{P_{i}\right\}$ are orthogonal projectors. Then after measurement one is left with

$$
\sum_{i} P_{i} \mathcal{F}_{2} \rho^{\otimes 2} \mathcal{F}_{2}^{\dagger} P_{i}
$$


(4) Measure $\mathcal{R}_{2}=\Pi_{+}-\Pi_{-}$(where $\Pi_{ \pm}$is the projector onto the \pm eigenspace) to obtain

$$
\sum_{i} \Pi_{+} P_{i} \mathcal{F}_{2} \rho^{\otimes 2} \mathcal{F}_{2}^{\dagger} P_{i} \Pi_{+}+\sum_{i} \Pi_{-} \mathcal{F}_{2} P_{i} \rho^{\otimes 2} P_{i} \mathcal{F}_{2}^{\dagger} \Pi_{-} .
$$

(5) The probability that one measures $\mathcal{R}_{2}$ as +1 , after having measured $\rho^{\otimes 2}$ to be in the subspace corresponding to $P_{i}$, is denoted by $\operatorname{Prob}(+\mid i)$. Similarly, the probability that one measures $\mathcal{R}_{2}$ as -1 , after having measured $\rho^{\otimes 2}$ to be in the subspace corresponding to $P_{i}$, is denoted by $\operatorname{Prob}(-\mid i)$. After obtaining $\operatorname{Prob}(+\mid i)$ and $\operatorname{Prob}(-\mid i)$, one can compute

$$
\begin{aligned}
\sum_{i} \lambda_{i}[\operatorname{Prob}(+\mid i)-\operatorname{Prob}(-\mid i)] & =\sum_{i} \lambda_{i} \operatorname{tr}\left\{\Pi_{+} P_{i} \mathcal{F}_{2} \rho^{\otimes 2} \mathcal{F}_{2}^{\dagger} P_{i} \Pi_{+}-\Pi_{-} \mathcal{F}_{2} P_{i} \rho^{\otimes 2} P_{i} \mathcal{F}_{2}^{\dagger} \Pi_{-}\right\} \\
& =\sum_{i} \lambda_{i} \operatorname{tr}\left\{\mathcal{R}_{2} P_{i} \mathcal{F}_{2} \rho^{\otimes 2} \mathcal{F}_{2}^{\dagger} P_{i}\right\} \\
& =\operatorname{tr}\left\{\mathcal{R}_{2} \mathcal{X}_{2} \mathcal{F}_{2} \rho^{\otimes 2} \mathcal{F}_{2}^{\dagger}\right\} \\
& =\operatorname{tr}\left\{X \rho^{2}\right\}
\end{aligned}
$$

where we have used $\left[\mathcal{X}_{2}, \mathcal{R}_{2}\right]=0$ to go from the second line to the third line, and Eq. (A6) to go from the third line to the last line. A similar procedure can be used to determine $\operatorname{tr}\left\{\rho^{2}\right\}$, and then one can compute the quotient $\operatorname{tr}\left\{X \rho^{2}\right\} / \operatorname{tr}\left\{\rho^{2}\right\}$.

In an actual experiment, one does not directly measure the parity operator $\mathcal{R}_{2}$, but instead measures the number operator on every site. Since the common refinement of the eigenspaces of all number operators is a refinement of the eigenspaces of $\mathcal{R}_{2}$, one can measure $\mathcal{R}_{2}$ via the number operators and obtain the same result as above.

\section{Fermion interferometry}

It is straightforward to adapt the boson interferometry techniques to fermions, although a few modifications to the protocol are required. Our protocol is inspired by the work of Ref. [41]. Suppose we have two systems of fermions, and require that states of different fermion number lie in different superselection sectors. Technically, the superselection rule means that for all observables $X$, we have $\left\langle\psi_{1}|X| \psi_{2}\right\rangle=0$ if $\left|\psi_{1}\right\rangle$ and $\left|\psi_{2}\right\rangle$ are states of definite, but distinct fermion number.

For fermions, it is not true that $\operatorname{tr}\left\{\mathcal{R}_{2} \mathcal{F}_{2} \rho^{\otimes 2} \mathcal{F}_{2}^{\dagger}\right\}=$ $\operatorname{tr}\left\{\rho^{2}\right\}$. Instead, we have

$$
\operatorname{tr}\left\{\mathcal{V F}_{2} \rho^{\otimes 2} \mathcal{F}_{2}^{\dagger}\right\}=\operatorname{tr}\left\{\rho^{2}\right\},
$$

where $\mathcal{V}$ has eigenvalues \pm 1 which depend on the total number of fermions $N_{\text {tot }}$, the floor of half of the total number of fermions $\left\lfloor N_{\text {tot }} / 2\right\rfloor$, and the number of fermions $\mathrm{N}_{2}$ in the second copy of the subsystem. [There are, in fact, many choices of $\mathcal{V}$ which satisfy Eq. (A12), and so we choose a convenient one for our purposes.] The measurement outcomes for $\mathcal{V}$ are given in Table I.

The procedure for measuring $\operatorname{tr}\left\{\mathcal{X}_{2} \rho^{2}\right\} / \operatorname{tr}\left\{\rho^{2}\right\}$ is the same as in the bosonic case above, except that now we need

$$
\mathcal{X}_{2}=\mathcal{F}_{2}\left[\frac{1}{2} X\left(\left\{f_{i, 1}, f_{i, 1}^{\dagger}\right\}\right)+\frac{1}{2} X\left(\left\{f_{i, 2}, f_{i, 2}^{\dagger}\right\}\right)\right] \mathcal{F}_{2}^{\dagger}
$$

(where here the $f, f^{\dagger}$ operators are fermionic) to additionally satisfy

$$
\left[\mathcal{X}_{2}, \mathcal{V}\right]=0
$$

So first let us find which operators, in general, commute with $\mathcal{V}$. Suppose we have an operator of the form

$$
\underbrace{f_{i_{1}, 1}^{\dagger} \ldots f_{i_{m_{1}}, 1}^{\dagger}}_{m_{1} \text { of these }} \underbrace{f_{j_{1}, 1} \ldots f_{j_{m_{2}}, 1}}_{m_{2} \text { of these }} \underbrace{f_{k_{1}, 2}^{\dagger} \ldots f_{k_{n_{1}}, 2}^{\dagger}}_{n_{1} \text { of these }} \underbrace{f_{\ell_{1}, 2 \ldots f_{\ell_{n_{2}, 2}}}}_{n_{2} \text { of these }},
$$

where $\left\{i_{1}, \ldots, i_{m_{1}}\right\},\left\{j_{1}, \ldots, j_{m_{2}}\right\},\left\{k_{1}, \ldots, k_{n_{1}}\right\},\left\{\ell_{1}, \ldots, \ell_{n_{2}}\right\}$ are all sets with nonrepeating elements. All of these operators transform multiplicatively by either +1 or -1 after conjugation by $\mathcal{V}$. Letting $m=\left|m_{1}-m_{2}\right|$ and $n=\left|n_{1}-n_{2}\right|$, the possibilities are presented in Table II.

For example, letting $\mathcal{X}_{2}=\frac{1}{2}\left(n_{i, 1}+n_{i, 2}\right)$, we have $\left[\mathcal{X}_{2}, \mathcal{V}\right]=0$. If instead $\mathcal{X}_{2}=\mathcal{F}_{2}\left(\frac{1}{2}\left(n_{i, 1} n_{j, 1}+n_{i, 2} n_{j, 2}\right) \mathcal{F}_{2}^{\dagger}\right.$, we likewise have $\left[\mathcal{X}_{2}, \mathcal{V}\right]=0$.

TABLE I. Characterization of measurement outcomes for $\mathcal{V}$.

\begin{tabular}{lccc}
\hline \hline$N_{\text {tot }}$ & $\left\lfloor N_{\text {tot }} / 2\right\rfloor$ & $N_{2}$ & Result \\
\hline Even & Even & Even & +1 \\
Even & Even & Odd & -1 \\
Even & Odd & Even & -1 \\
Even & Odd & Odd & +1 \\
Odd & Even & Even & +1 \\
Odd & Even & Odd & -1 \\
Odd & Odd & Even & -1 \\
Odd & Odd & Odd & +1 \\
\hline \hline
\end{tabular}


TABLE II. Transformation of products of fermion operators under conjugation by $\mathcal{V}$.

\begin{tabular}{lccc}
\hline \hline$m+n(\bmod 2)$ & $m+n(\bmod 4)$ & $n(\bmod 2)$ & Result \\
\hline 0 & 2 & 0 & -1 \\
0 & 2 & 1 & +1 \\
0 & 0 & 0 & +1 \\
0 & 0 & 1 & -1 \\
1 & 1 & 0 & -1 \\
1 & 1 & 1 & +1 \\
1 & 3 & 0 & +1 \\
1 & 3 & 1 & -1 \\
\hline \hline
\end{tabular}

\section{APPENDIX B: EXTRACTING TWO-POINT CORRELATIONS IN THE LOW-TEMPERATURE LIMIT}

In this Appendix, we numerically study the effect of the second term in Eq. (5) in the main text. More specifically, we have argued that one can extract a density-density correlation from a more experimentally accessible quantity:

$C(j, \ell) \equiv \frac{1}{2} \operatorname{tr}\left\{n_{j} n_{\ell} \rho(T / 2)\right\}+\frac{1}{2} \frac{\operatorname{tr}\left\{n_{j} \rho(T) n_{\ell} \rho(T)\right\}}{\operatorname{tr}\left\{\rho(T)^{2}\right\}}$.

While the first term is the desired density-density correlation, the second term arises as a consequence of the Fourier transform of local operators $n_{j}$ and $n_{\ell}$. As described in the main text, however, we expect that at sufficiently low temperatures the second term does not exhibit any systematic dependence on the distance between two points $d=|j-\ell|$, allowing us to extract physically meaningful quantities such as correlations lengths from fitting $C(j, \ell)$ as a function of $d$.

In order to confirm this expectation, we consider a 1D Bose-Hubbard Hamiltonian with nearest-neighbor hopping rate $J$ and on-site repulsive interaction $U=3 J$. We numerically compute thermal density matrices for $N=4$ particles on $L=16$ lattice sites with periodic boundary condition at various temperature, $T / J \in\left\{\frac{1}{10}, \frac{1}{5}, \frac{1}{4}, \frac{1}{2}, 1\right\}$. For each temperature $T$, we compute each term in $C(j, \ell)$ as well as their sum as a function of the distance $d \in\{1, \ldots, 8\}$. Figure 4 summarizes our numerical results, from which it can be checked that the density-density correlation [the first term in $C(j, \ell)$ ] displays strong antibunching [Fig. 4(a)] at low temperature. By contrast, the second term exhibits diminishing distance dependence as the temperature decreases [Fig. 4(b)]. We find that the distance dependence of the total value $C(j, \ell)$ is indeed dominated by the density-density correlation [Fig. 4(c)] at sufficiently low temperatures.

\section{APPENDIX C: EXPERIMENTAL METHODS}

Our experiments start from a high-fidelity Mott insulator with a single particle per lattice site. Using high-precision, site-resolved optical potentials, created by a digital micromirror device (DMD), we isolate two neighboring six-site chains of atoms with exactly one atom on each site. In order to ensure the high fidelity of the initial state, we hold it in the $45 E_{r}$ deep optical lattice in both directions. To obtain a locally thermal state, we suddenly drop the lattice depth along the chains, allowing atoms to tunnel, while keeping the lattice high between the chains. We use a pair of DMD beams to offset the sites right outside the region of interest, thereby defining the overall length of the system. After variable evolution time, we freeze the dynamics along the chains by suddenly ramping up the lattice back to $45 E_{r}$. In order to make sure that the state has thermalized, we pick evolution times for which the entanglement entropy of the region of interest has reached its saturation value. Table III shows the times used in Fig. 2 in the main text for each case studied. (a)

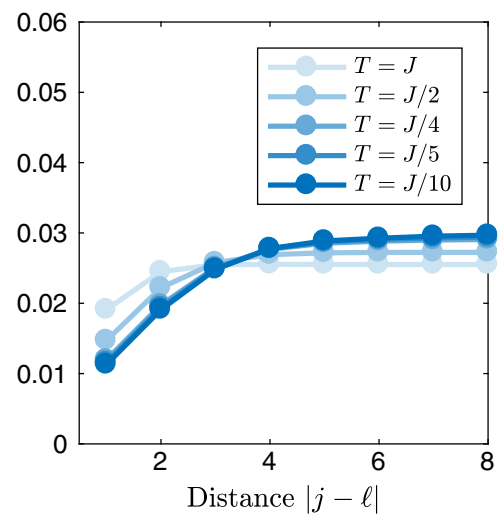

(b)

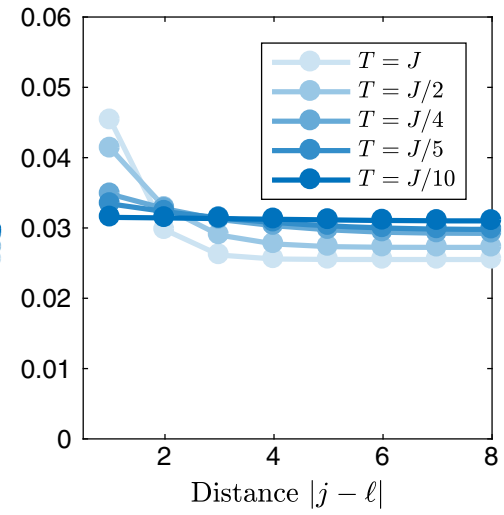

(c)

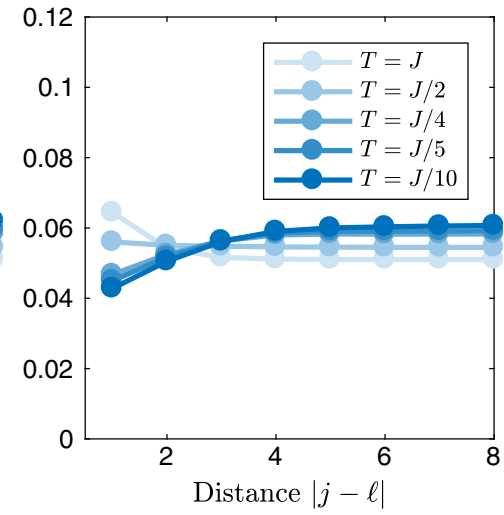

FIG. 4. Extracting the density-density correlation from Eq. (5) in the main text. (a) Density-density correlations in a 1D Bose-Hubbard model at various temperatures. This quantity corresponds to the first term in $C(j, \ell)$. (b) Additional contribution to $C(j, \ell)$ arising from the second term. Crucially, this contribution exhibits decreasing distance dependence in the low-temperature limit. (c) The position dependence of the total value $C(j, \ell)$ is dominated by the first term in low-temperature limit. 
TABLE III. Evolution times used for each case in Fig. 2 of main text.

\begin{tabular}{lc}
\hline \hline Case & Times $(\hbar / J)$ \\
\hline $\mathrm{A}$ & $1.0,1.4,2.2,4.3,5.1,6.4,8.4$ \\
$\mathrm{~B}$ & $12.2,24.0,59.4$ \\
$\mathrm{C}$ & $22.4,41.3$ \\
\hline \hline
\end{tabular}

In order to implement the beam splitter operation, we drop the lattice depth between the chains and let the atoms evolve for a certain time duration. During this process the lattice depth along the chains stays high, preventing wave function evolution in that direction. At the end of this sequence, we read out the state of the system in the particle number basis with single-site and full-atom-number resolution. For more details, see Ref. [28].

[1] S. Lloyd, Universal Quantum Simulators, Science 273, 1073 (1996).

[2] C. Gross and I. Bloch, Quantum Simulations with Ultracold Atoms in Optical Lattices, Science 357, 995 (2017).

[3] I. M. Georgescu, S. Ashhab, and F. Nori, Quantum Simulation, Rev. Mod. Phys. 86, 153 (2014).

[4] P. Jurcevic, B. P. Lanyon, P. Hauke, C. Hempel, P. Zoller, R. Blatt, and C. F. Roos, Quasiparticle Engineering and Entanglement Propagation in a Quantum Many-Body System, Nature (London) 511, 202 (2014).

[5] J. Zhang, G. Pagano, P. W. Hess, A. Kyprianidis, P. Becker, H. Kaplan, A. V. Gorshkov, Z.-X. Gong, and C. Monroe, Observation of a Many-Body Dynamical Phase Transition with a 53-Qubit Quantum Simulator, Nature (London) 551, 601 (2017).

[6] E. Guardado-Sanchez, P. T. Brown, D. Mitra, T. Devakul, D. A. Huse, P. Schauß, and W. S. Bakr, Probing the Quench Dynamics of Antiferromagnetic Correlations in a 2D Quantum Ising Spin System, Phys. Rev. X 8, 021069 (2018).

[7] V. Lienhard, S. de Léséleuc, D. Barredo, T. Lahaye, A. Browaeys, M. Schuler, L.-P. Henry, and A. M. Läuchli, Observing the Space-and Time-Dependent Growth of Correlations in Dynamically Tuned Synthetic Ising Models with Antiferromagnetic Interactions, Phys. Rev. X 8, 021070 (2018).

[8] H. Bernien et al., Probing Many-Body Dynamics on a 51Atom Quantum Simulator, Nature (London) 551, 579 (2017).

[9] R. Barends et al., Digitized Adiabatic Quantum Computing with a Superconducting Circuit, Nature (London) 534, 222 (2016).

[10] C. Eichler, J. Mlynek, J. Butscher, P. Kurpiers, K. Hammerer, T. J. Osborne, and A. Wallraff, Exploring Interacting Quantum Many-Body Systems by Experimentally Creating Continuous Matrix Product States in Superconducting Circuits, Phys. Rev. X 5, 041044 (2015).

[11] M. H. Devoret and R. J. Schoelkopf, Superconducting Circuits for Quantum Information: An Outlook, Science 339, 1169 (2013).
[12] D. Jaksch, C. Bruder, J. I. Cirac, C. W. Gardiner, and P. Zoller, Cold Bosonic Atoms in Optical Lattices, Phys. Rev. Lett. 81, 3108 (1998).

[13] M. Greiner, O. Mandel, T. Esslinger, T. W. Hänsch, and I. Bloch, Quantum Phase Transition from a Superfluid to a Mott Insulator in a Gas of Ultracold Atoms, Nature (London) 415, 39 (2002).

[14] M. Köhl, H. Moritz, T. Stöferle, K. Günter, and T. Esslinger, Fermionic Atoms in a Three Dimensional Optical Lattice: Observing Fermi Surfaces, Dynamics, and Interactions, Phys. Rev. Lett. 94, 080403 (2005).

[15] M. Aidelsburger, M. Atala, M. Lohse, J. T. Barreiro, B. Paredes, and I. Bloch, Realization of the Hofstadter Hamiltonian with Ultracold Atoms in Optical Lattices, Phys. Rev. Lett. 111, 185301 (2013).

[16] H. Miyake, G. A. Siviloglou, C. J. Kennedy, W. C. Burton, and W. Ketterle, Realizing the Harper Hamiltonian with Laser-Assisted Tunneling in Optical Lattices, Phys. Rev. Lett. 111, 185302 (2013).

[17] B. K. Stuhl, H.-I. Lu, L. M. Aycock, D. Genkina, and I. B. Spielman, Visualizing Edge States with an Atomic Bose Gas in the Quantum Hall Regime, Science 349, 1514 (2015).

[18] M. Mancini et al., Observation of Chiral Edge States with Neutral Fermions in Synthetic Hall Ribbons, Science 349, 1510 (2015).

[19] S. Baier, M. J. Mark, D. Petter, K. Aikawa, L. Chomaz, Z. Cai, M. Baranov, P. Zoller, and F. Ferlaino, Extended BoseHubbard Models with Ultracold Magnetic Atoms, Science 352, 201 (2016).

[20] J. Simon, W. S. Bakr, R. Ma, M. E. Tai, P. M. Preiss, and M. Greiner, Quantum Simulation of Antiferromagnetic Spin Chains in an Optical Lattice, Nature (London) 472, 307 (2011).

[21] D. Greif, T. Uehlinger, G. Jotzu, L. Tarruell, and T. Esslinger, Short-Range Quantum Magnetism of Ultracold Fermions in an Optical Lattice, Science 340, 1307 (2013).

[22] R. A. Hart, P. M. Duarte, T.-L. Yang, X. Liu, T. Paiva, E. Khatami, R. T. Scalettar, N. Trivedi, D. A. Huse, and R. G. Hulet, Observation of Antiferromagnetic Correlations in the Hubbard Model with Ultracold Atoms, Nature (London) 519, 211 (2015).

[23] M. F. Parsons, A. Mazurenko, C. S. Chiu, G. Ji, D. Greif, and M. Grein, Site-Resolved Measurement of the SpinCorrelation Function in the Fermi-Hubbard Model, Science 353, 1253 (2016).

[24] M. Boll, T. A. Hilker, G. Salomon, A. Omran, J. Nespolo, L. Pollet, I. Bloch, and C. Gross, Spin- and Density-Resolved Microscopy of Antiferromagnetic Correlations in FermiHubbard Chains, Science 353, 1257 (2016).

[25] L. W. Cheuk, M. A. Nichols, K. R. Lawrence, M. Okan, H. Zhang, E. Khatami, N. Trivedi, T. Paiva, M. Rigol, and M. W. Zwierlein, Observation of Spatial Charge and Spin Correlations in the 2D Fermi-Hubbard Model, Science 353, 1260 (2016).

[26] A. Mazurenko, C. S. Chiu, G. Ji, M. F. Parsons, M. KanászNagy, R. Schmidt, F. Grusdt, E. Demler, D. Greif, and M. Greiner, A Cold-Atom Fermi-Hubbard Antiferromagnet, Nature (London) 545, 462 (2017).

[27] R. Islam, R. Ma, P. M. Preiss, M. E. Tai, A. Lukin, M. Rispoli, and M. Greiner, Measuring Entanglement Entropy 
in a Quantum Many-Body System, Nature (London) 528, 77 (2015).

[28] A. M. Kaufman, M. E. Tai, A. Lukin, M. Rispoli, R. Schittko, P. M. Preiss, and M. Greiner, Quantum Thermalization through Entanglement in an Isolated Many-Body System, Science 353, 794 (2016).

[29] T. Brydges et al., Probing Entanglement Entropy via Randomized Measurements, Science 364, 260 (2019).

[30] M. Cheneau, P. Barmettler, D. Poletti, M. Endres, P. Schauß, T. Fukuhara, C. Gross, I. Bloch, C. Kollath, and S. Kuhr, Light-Cone-like Spreading of Correlations in a Quantum Many-Body System, Nature (London) 481, 484 (2012).

[31] F. Meinert, M. J. Mark, E. Kirilov, K. Lauber, P. Weinmann, M. Grobner, A. J. Daley, and H.-C. Nagerl, Observation of Many-Body Dynamics in Long-Range Tunneling after a Quantum Quench, Science 344, 1259 (2014).

[32] M. Schreiber, S. S. Hodgman, P. Bordia, H. P. Luschen, M. H. Fischer, R. Vosk, E. Altman, U. Schneider, and I. Bloch, Observation of Many-Body Localization of Interacting Fermions in a Quasi-Random Optical Lattice, Science 349, 842 (2015).

[33] L. Alexander et al., Probing Entanglement in a Many-BodyLocalized System, Science 364, 256 (2019).

[34] C. Chu, G. Ji, A. Mazurenko, D. Greif, and M. Greiner, Quantum State Engineering of a Hubbard System with Ultracold Fermions, Phys. Rev. Lett. 120, 243201 (2018).

[35] T.-L. Ho, and Q. Zhou, Squeezing Out the Entropy of Fermions in Optical Lattices, Proc. Natl. Acad. Sci. U.S.A. 106, 6916 (2009).

[36] A. Kantian, S. Langer, and A. J. Daley, Dynamical Disentangling and Cooling of Atoms in Bilayer Optical Lattices, Phys. Rev. Lett. 120, 060401 (2018).

[37] A. K. Ekert, C. M. Alves, D. K. L. Oi, M. Horodecki, P. Horodecki, and L. C. Kwek, Direct Estimations of Linear and Nonlinear Functionals of a Quantum State, Phys. Rev. Lett. 88, 217901 (2002).

[38] T. A. Brun, Measuring Polynomial Functions of States, Quantum Inf. Comput. 4, 401 (2004).

[39] C. M. Alves and D. Jaksch, Multipartite Entanglement Detection in Bosons, Phys. Rev. Lett. 93, 110501 (2004).

[40] A. J. Daley, H. Pichler, J. Schachenmayer, and P. Zoller, Measuring Entanglement Growth in Quench Dynamics of Bosons in an Optical Lattice, Phys. Rev. Lett. 109, 020505 (2012).
[41] H. Pichler, L. Bonnes, A. J. Daley, A. M. Läuchli, and P. Zoller, Thermal versus Entanglement Entropy: A Measurement Protocol for Fermionic Atoms with a Quantum Gas Microscope, New J. Phys. 15, 063003 (2013).

[42] J. M. Deutsch, Quantum Statistical Mechanics in a Closed System, Phys. Rev. A 43, 2046 (1991).

[43] M. Srednicki, Chaos and Quantum Thermalization, Phys. Rev. E 50, 888 (1994).

[44] M. Srednicki, The Approach to Thermal Equilibrium in Quantized Chaotic Systems, J. Phys. A 32, 1163 (1999).

[45] M. Rigol, V. Dunjko, and M. Olshanii, Thermalization and Its Mechanism for Generic Isolated Quantum Systems, Nature (London) 452, 854 (2008).

[46] L. D'Alessio, Y. Kafri, A. Polkovnikov, and M. Rigol, From Quantum Chaos and Eigenstate Thermalization to Statistical Mechanics and Thermodynamics, Adv. Phys. 65, 239 (2016).

[47] R. R. P. Singh, M. B. Hastings, A. B. Kallin, and R. G. Melko, Finite-Temperature Critical Behavior of Mutual Information, Phys. Rev. Lett. 106, 135701 (2011).

[48] J. R. Garrison and T. Grover, Does a Single Eigenstate Encode the Full Hamiltonian?, Phys. Rev. X 8, 021026 (2018).

[49] Note that a particle number superselection rule is required for Eq. (2).

[50] H. Pichler, G. Zhu, A. Seif, P. Zoller, and M. Hafezi, Measurement Protocol for the Entanglement Spectrum of Cold Atoms, Phys. Rev. X 6, 041033 (2016).

[51] D. Jaksch and P. Zoller, Creation of effective magnetic fields in optical lattices: the Hofstadter butterfly for cold neutral atoms, New J. Phys. 5, 56 (2003).

[52] L.-M. Duan, M. D. Lukin, J. I. Cirac, and P. Zoller, Long-Distance Quantum Communication with Atomic Ensembles and Linear Optics, Nature (London) 414, 413 (2001).

[53] L. F. Santos, A. Polkovnikov, and M. Rigol, Weak and Strong Typicality in Quantum Systems, Phys. Rev. E 86, 010102(R) (2012).

[54] J. M. Deutsch, H. Li, and A. Sharma, Microscopic Origin of Thermodynamic Entropy in Isolated Systems, Phys. Rev. E 87, 042135 (2013).

[55] K. R. Fratus and S. V. Truong, Does a Single Eigenstate of a Hamiltonian Encode the Critical Behaviour of Its FiniteTemperature Phase Transition?, arXiv:1810.11092. 